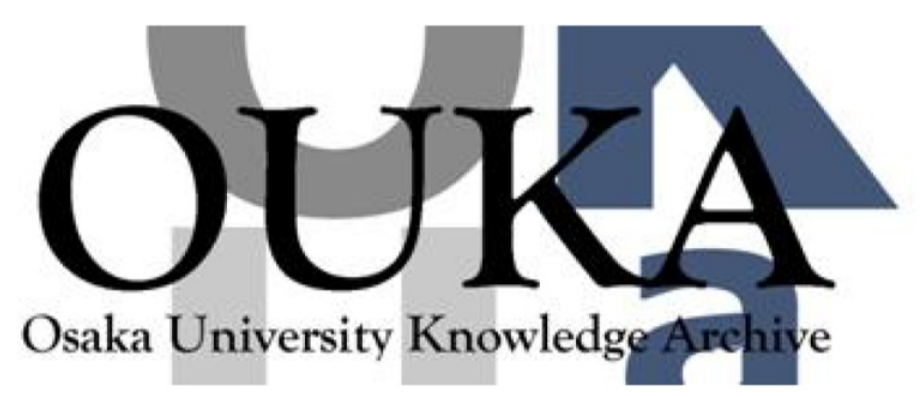

\begin{tabular}{|c|c|}
\hline Title & $\begin{array}{l}\text { Thermodynamics of strongly-coupled Yukawa } \\
\text { systems near the one-component-plasma Limit. } \\
\text { II. Molecular dynamics simulations }\end{array}$ \\
\hline Author (s) & Farouki, R. T.; Hamaguchi, S. \\
\hline Citation & $\begin{array}{l}\text { The Journal of Chemical Physics. 101(11) } \\
\text { p. 9885-p. } 9893\end{array}$ \\
\hline Issue Date & $1994-12-01$ \\
\hline oaire:version & VoR \\
\hline URL & https://hdl. handle. net/11094/78504 \\
\hline rights & $\begin{array}{l}\text { This article may be downloaded for personal use } \\
\text { only. Any other use requires prior permission } \\
\text { of the author and AIP Publishing. This article } \\
\text { appeared in J. Chem. Phys. 101, 9885 (1994) and } \\
\text { may be found at } \\
\text { https://doi.org/10.1063/1.467955. }\end{array}$ \\
\hline Note & \\
\hline
\end{tabular}

Osaka University Knowledge Archive : OUKA

https://ir. Library. osaka-u. ac. jp/

Osaka University 


\section{Thermodynamics of strongly-coupled Yukawa systems near the one- component-plasma limit. II. Molecular dynamics simulations}

Cite as: J. Chem. Phys. 101, 9885 (1994); https://doi.org/10.1063/1.467955

Submitted: 02 May 1994 . Accepted: 31 August 1994 . Published Online: 31 August 1998

R. T. Farouki, and S. Hamaguchi

\section{ARTICLES YOU MAY BE INTERESTED IN}

Thermodynamics of strongly-coupled Yukawa systems near the one-componentplasma limit. I. Derivation of the excess energy

The Journal of Chemical Physics 101, 9876 (1994); https://doi.org/10.1063/1.467954

Phase diagram of Yukawa systems near the one-component-plasma limit revisited The Journal of Chemical Physics 105, 7641 (1996); https://doi.org/10.1063/1.472802

Phase diagram and dynamics of Yukawa systems

The Journal of Chemical Physics 88, 3286 (1988); https://doi.org/10.1063/1.453924

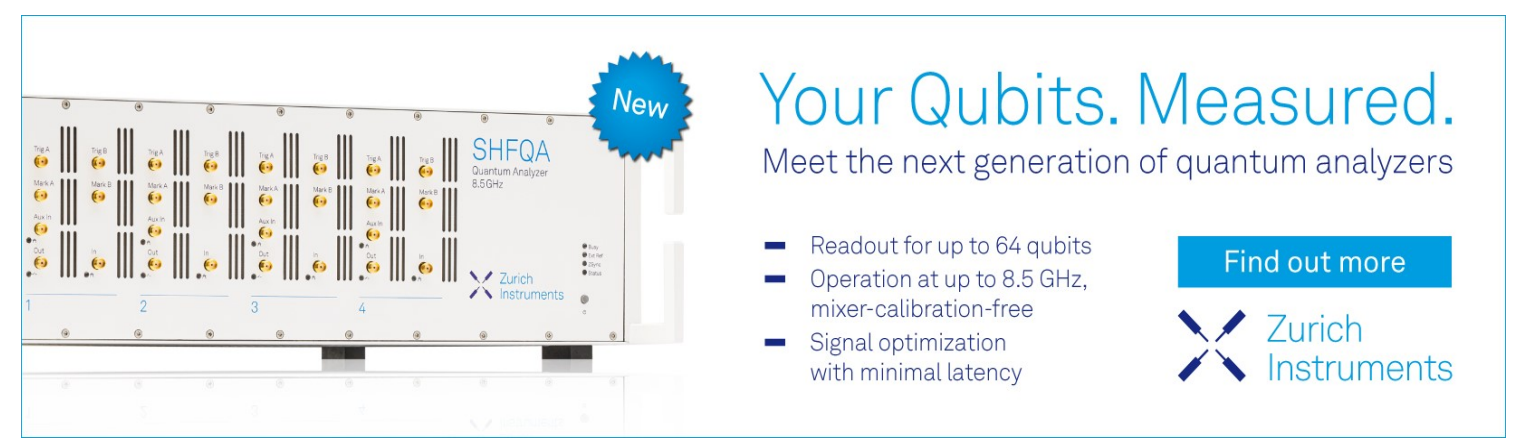




\title{
Thermodynamics of strongly-coupled Yukawa systems near the one-component-plasma limit. II. Molecular dynamics simulations
}

\author{
R. T. Farouki and S. Hamaguchi \\ IBM Thomas J. Watson Research Center, P. O. Box 218, Yorktown Heights, New York 10598
}

(Received 2 May 1994; accepted 31 August 1994)

\begin{abstract}
Molecular dynamics simulations are employed to study the equilibrium thermodynamics of strongly-coupled systems of particles interacting through the Yukawa potential. Such systems serve, under the Debye-Hückel approximation, as a model for the physical behavior of plasma or colloidal suspensions of charged particulates. The thermodynamics may be characterized in terms of two dimensionless parameters - the ratio $\kappa$ of the mean interparticle distance to the Debye length, and an approximate measure $\Gamma$ of the interparticle potential energy in units of the thermal kinetic energy. Employing an accurate representation of infinite periodic boundary conditions, we focus on the regime of weak Debye screening $(\kappa \leqslant 1)$ and strong coupling $(\Gamma \gg 1)$. Excess internal energies measured at many points $(\kappa, \Gamma)$ are fitted to simple functional forms for the fluid and solid phases, representing extrapolations of the classical one-component plasma (OCP) limit, $\kappa=0$. Quantitative expressions for the Helmholtz free energy and the "equation of state" of the Yukawa systemgiving the pressure $p$ in terms of $\kappa$ and $\Gamma$-are thus derived, and the freezing/melting curve in the $(\kappa, \Gamma)$ plane is traced as the intersection of the fluid and solid free-energy surfaces. (C) 1994 American Institute of Physics.
\end{abstract}

\section{INTRODUCTION}

Particulate contamination in microelectronics process plasmas is currently attracting much attention. ${ }^{1-4}$. The macroscopic behavior of suspensions of contaminant particles in such plasmas is complicated by the fact that, under representative conditions, the particulates may acquire large negative charges and behave as a "strongly coupled" system, ${ }^{5-7}$ i.e., the potential energy of the (screened) Coulomb interactions of particulates can exceed their thermal kinetic energy by a large factor. Strongly-coupled systems arise in a variety of physical contexts, e.g., colloidal suspensions, ${ }^{8}$ dust grains in planetary magnetospheres, ${ }^{9}$ and the classical "onecomponent plasma" $(\mathrm{OCP})^{10}$ - a model for the interior of white dwarf stars.

Consider a system of identical particulates of mass $m$, charge $Q=-Z e(Z \gg 1)$, density $n$, and temperature $T$, immersed in a neutralizing background plasma of fixed mean temperature and density. The pair potential that describes the interaction of two particulates is the Yukawa form: ${ }^{11}$

$$
\phi(r)=\frac{Q^{2}}{4 \pi \epsilon_{0} r} \exp \left(-k_{D} r\right),
$$

where $k_{D}$ is the inverse of the Debye length

$$
\lambda_{D}=\left(\frac{q_{i}^{2} \bar{n}_{i}}{\epsilon_{0} k T_{i}}+\frac{e^{2} \bar{n}_{e}}{\epsilon_{0} k T_{e}}\right)^{-1 / 2},
$$

$q_{i}, \bar{n}_{i}$, and $T_{i}$ being the charge, mean density, and temperature of the plasma ions, and $-e, \bar{n}_{e}$, and $T_{e}$ the corresponding quantities for plasma electrons $\left[q_{i} \vec{n}_{i}=e\left(\vec{n}_{e}+Z n\right)\right.$ for overall charge neutrality]. We assume that $m$ is much larger than atomic masses, so the background plasma may be regarded as instantaneously relaxing to form a spherical Debye sheath around each particulate on time-scales characterizing the particulate motions.
It is convenient to measure interparticle distances in units of the Wigner-Seitz radius $a=(3 / 4 \pi n)^{1 / 3}$. The thermodynamics of the particulate system may then be described in terms of two dimensionless ratios:

$$
\kappa=\frac{a}{\lambda_{D}} \text { and } \Gamma=\frac{Q^{2}}{4 \pi \epsilon_{0} a k T} .
$$

The parameter $\kappa$ gives an indication of the importance of Debye screening, while the "coupling" parameter $\Gamma$ is roughly the ratio of the (unscreened) Coulomb potential energy to the thermal kinetic energy per particle.

Our goal is to develop quantitative thermodynamic models for the Yukawa system, based on molecular dynamics simulations, in the regime of strong coupling $(\Gamma \gg 1$, where the system is far from ideal-gas behavior) and weak Debye screening $(\kappa \leqslant 1)$. Although there have been many studies $^{12-19}$ of Yukawa systems, using both molecular dynamics and Monte Carlo methods, they have focused mostly on the case of strong screening $(\kappa>1)$, where the simple "minimum image" method ${ }^{20}$ provides a fair approximation to infinite periodic boundary conditions. This method does not yield accurate internal energy measurements if $\kappa \leqslant 1$, however, since the pair potential (1) must then be augmented by a term that accurately reflects the interaction of one particle with all periodic images of another particle. ${ }^{21}$

Another reason for concentrating on the weak-screening regime is that the OCP - a system of mobile charges immersed in a smooth, strictly uniform neutralizing background -is the formal limit $\kappa \rightarrow 0$ of the Yukawa system. The OCP thermodynamics has been extensively studied on a quantitative basis, ${ }^{22-32}$ and simple functional forms are available that describe the free energy of its fluid and solid phases in terms of $\Gamma$ to high accuracy. Thus, we can readily construct our thermodynamic model of the Yukawa system as an extrapolation of established OCP properties to small but finite $\kappa$. 


\section{MOLECULAR DYNAMICS SIMULATIONS}

For periodic boundary conditions appropriate to a cubical simulation volume of side $L$, we use in lieu of (1) the "effective" pair potential describing the interaction of particle $k$ with particle $j$ (at separation $\mathbf{r}=\mathbf{r}_{k}-\mathbf{r}_{j}$ ) and with all periodic images of the latter,

$$
\Phi(\mathbf{r})=\phi(|\mathbf{r}|)+\sum_{\mathbf{n} \neq \mathbf{0}} \phi(|\mathbf{r}+\mathbf{n} L|)
$$

where the infinite sum is over vectors $\mathbf{n}=(l, m, n)$ with integer components. This cubically-symmetric term is approximated by a tensor-product spline function interpolating an array of $40 \times 40 \times 40$ discrete values, summed to high accuracy. The approximation can be efficiently evaluated in the simulations, and has a fractional deviation from the exact value of no more than $\sim 10^{-7}$. Full details of the approximation scheme may be found in Ref. 21.

As units of mass, length, and time, we employ the particulate mass $m$, the Wigner-Seitz radius $a$, and $\sqrt{3} \omega_{\mathrm{p}}^{-1}$ where $\omega_{\mathrm{p}}=\sqrt{Q^{2} n / \epsilon_{0} m}$ is the plasma frequency for the particulates. The equations of motion are then

$$
\frac{d^{2} \xi_{k}}{d \tau^{2}}=-\sum_{j \neq k}^{N} \nabla \hat{\Phi}\left(\xi_{k}-\xi_{j}\right) \quad \text { for } \quad k=1, \ldots, N
$$

where $\boldsymbol{\xi}_{k}=\mathbf{r}_{k} / a$ is the dimensionless location of particle $k$, $\tau=\omega_{\mathrm{p}} t / \sqrt{3}$ is the dimensionless time, and $\hat{\Phi}=4 \pi \epsilon_{0} a \Phi / Q^{2}$ is the dimensionless" "effective" pair potential. We integrate the system (5) by a predictor-corrector scheme in which the variation of the force on a particle over each timestep is obtained by extrapolation from a quadratic fitting polynomial that interpolates its value at three preceding instances; the "correction" stage adds a cubic term. Forces are obtained by pairwise summation over all particles -i.e., there is no cutoff radius - and the integration proceeds on an asynchronous timefront using individually-adjusted steps to ensure uniformly accurate trajectories under varying conditions.

To emulate average system properties for a canonical ensemble with fixed $\Gamma$ (temperature), we periodically synchronize all particles and renormalize their velocities to the prescribed target value for $\Gamma$. Empirically, $\Delta \tau=1$ was determined to be the optimum interval for evolution of the system between temperature adjustments; measurements of thermodynamic properties were essentially unchanged upon adopting smaller integration periods. Typically, the total system energy was conserved to a fractional accuracy of $10^{-5}$ to $10^{-6}$ over each integration period.

In the case $\kappa=0$ the infinite sum in (4) does not converge, and this effective potential must be replaced by the well-known Ewald potential for the OCP. In dimensionless form, the Ewald potential is given ${ }^{22}$ by

$$
\begin{aligned}
\hat{\Phi}_{\mathrm{OCP}}(\xi)= & \frac{\operatorname{erfc}(\sqrt{\pi} \xi / \Lambda)}{\xi}-\frac{1}{\Lambda}+\sum_{\mathbf{n} \neq \mathbf{0}}\left[\frac{\operatorname{erfc}(\sqrt{\pi}|\xi+\mathbf{n} \Lambda| / \Lambda)}{|\boldsymbol{\xi}+\mathbf{n} \Lambda|}\right. \\
& \left.+\frac{\exp \left(-\pi|\mathbf{n}|^{2}\right) \cos (2 \pi \mathbf{n} \cdot \xi / \Lambda)}{\pi|\mathbf{n}|^{2} \Lambda}\right]
\end{aligned}
$$

where $\boldsymbol{\xi}=\boldsymbol{\xi}_{k}-\boldsymbol{\xi}_{j}$ denotes the position of particle $k$ relative to particle $j, \operatorname{erfc}(\cdot)$ is the complementary error function, and $\Lambda=L / a=(4 \pi N / 3)^{1 / 3}$ is the size of the cubical simulation volume. The infinite sum in (6) is approximated in the same manner as that in (4)-unlike (4), however, the sum in (6) should not be interpreted as simply representing the effect of "image" particles.

The simulations commenced with particles positioned on a bcc lattice, which is the equilibrium configuration of the frozen Yukawa system for small $\kappa$-the bcc form has lower Madelung energy ${ }^{15,33-35}$ than the fcc form for $\kappa \leqslant 1.066$. To allow many runs at different $\kappa$ and $\Gamma$ values, we use $N=686$ (corresponding to a $7 \times 7 \times 7$ bcc lattice) for the simulations reported here. All runs were evolved for 300 time units, allowing the system to equilibriate to the desired $\Gamma$ for 100 units before averaging its properties over $100<\tau<300$. Cases that melted to a fluid state did so well before $\tau=100$.

As shown in the companion paper, ${ }^{36}$ the potential or "excess" energy per particle of the Yukawa system, including contributions of the background plasma, may be expressed in units of $k T$ as

$$
\begin{aligned}
\frac{U}{N k T}= & \Gamma\left[\frac{1}{N} \sum_{j=1}^{N-1} \sum_{k=j+1}^{N} \hat{\Phi}\left(\xi_{k}-\xi_{j}\right)-\frac{3}{2 \kappa^{2}}\right. \\
& \left.-\frac{\kappa}{2}+\frac{1}{2} \sum_{\mathbf{n} \neq \mathbf{0}} \frac{\exp (-\kappa|\mathbf{n}| \Lambda)}{|\mathbf{n}| \Lambda}\right]
\end{aligned}
$$

[this is the dimensionless form of equation (28) in Ref. 36]. The above expression is valid only for $\kappa>0$; for $\kappa=0$, we use: ${ }^{22}$

$$
\frac{U_{\mathrm{OCP}}}{N k T}=\Gamma\left[\frac{1}{N} \sum_{j=1}^{N-1} \sum_{k=j+1}^{N} \hat{\Phi}_{\mathrm{OCP}}\left(\boldsymbol{\xi}_{k}-\boldsymbol{\xi}_{j}\right)+\frac{E_{m}}{2}\right],
$$

where

$$
E_{m}=\lim _{\xi \rightarrow 0}\left(\hat{\Phi}_{\mathrm{OCP}}(\xi)-\frac{1}{\xi}\right)=\frac{-2.837297479}{\Lambda} .
$$

It may be verified ${ }^{36}$ that $U \rightarrow U_{\text {OCP }}$ as $\kappa \rightarrow 0$.

The inclusion of accurate periodic-correction terms in (4) and (6) allows energy data representative of infinite systems to be obtained with relatively small particle numbers. ${ }^{21}$ By way of empirical corroboration, we compare in Táble I energy measurements for the solid OCP phase from independent runs with $N=686$ and $N=1024$. The agreement is seen to be excellent, indicating that the measured energies are of much greater accuracy than the quoted rms thermal fluctuations seem to suggest (these fluctuations may be somewhat influenced by the adopted interval $\Delta \tau=1$ between tempera- 
TABLE I. OCP excess energy per particle $u / \Gamma$ obtained with $N=686$ and 1024.

\begin{tabular}{rcc}
\hline$\Gamma$ & $N=686$ & $N=1024$ \\
\hline 200 & $-0.887997 \pm 0.000195$ & $-0.888007 \pm 0.000163$ \\
240 & $-0.889426 \pm 0.000157$ & $-0.889421 \pm 0.000126$ \\
300 & $-0.890781 \pm 0.000124$ & $-0.890776 \pm 0.000101$ \\
400 & $-0.892098 \pm 0.000088$ & $-0.892097 \pm 0.000084$ \\
500 & $-0.892879 \pm 0.000079$ & $-0.892878 \pm 0.000059$ \\
600 & $-0.893396 \pm 0.000065$ & $-0.893395 \pm 0.000054$ \\
800 & $-0.894036 \pm 0.000050$ & $-0.894036 \pm 0.000041$ \\
1000 & $-0.894418 \pm 0.000040$ & $-0.894418 \pm 0.000040$ \\
\hline \hline
\end{tabular}

ture adjustments). Consequently, we shall use only unweighted least-squares fits to the measured energy data.

\section{THERMODYNAMIC PROPERTIES}

A wide range of physical conditions may be described by simple relations if we choose the dimensionless quantities $\kappa$ and $\Gamma$, in lieu of the particle density $n$ and temperature $T$, as independent thermodynamic variables:

However, we must first specify a model for the dependence of the Debye length $\lambda_{D}$, given by Eq. (2), upon $n$ and $T$. We shall assume that $T_{i}$ and $T_{e}$ are fixed and independent of $T$, while $\bar{n}_{i}$ and $\bar{n}_{e}$ are in constant proportion to $n$. The transformation of standard thermodynamic equations to dimensionless form is then governed by the relations

$$
\frac{\partial \kappa}{\partial n}=\frac{\kappa}{6 n}, \quad \frac{\partial \kappa}{\partial T}=0 \quad \text { and } \quad \frac{\partial \Gamma}{\partial n}=\frac{\Gamma}{3 n}, \quad \frac{\partial \Gamma}{\partial T}=-\frac{\Gamma}{T} .
$$

An extensive system comprising $N$ particles at temperature $T$ occupying a volume $V$ has Helmholtz free energy $F=U-T S$, where $U$ and $S$ denote the internal energy and entropy of the system. (Note that $F$ is the free energy with respect to the temperature $T$ of the particulates, it being understood that the background ion and electron temperatures are fixed. This $F$ should not be confused with the free energy with respect to the background temperatures, as discussed in Ref. 36.) If $F$ is known as a function of $T$ and $V$, the relations $P=-(\partial F / \partial V)_{T}$ and $S=-(\partial F / \partial T)_{V}$ give the pressure and entropy of the system. These relations can be recast in terms of dimensionless intensive variables as

$$
p=n\left(\frac{\partial f}{\partial n}\right)_{T} \text { and } s=-f-T\left(\frac{\partial f}{\partial T}\right)_{n},
$$

where $n=N / V$ is the particle number density, and

$u=\frac{U}{N k T}, f=\frac{F}{N k T}, p=\frac{P V}{N k T}$, and $s=\frac{S}{N k}$

denote the internal energy and free energy per particle in units of $k T$, the pressure in units of $n k T$, and the entropy per particle in units of $k$.

If we know the free energy as a function $f(\kappa, \Gamma)$ of the parameters (3), we can determine the pressure $p$ and entropy $s$ as functions of these variables by making use of (9) and the chain rule to rewrite expressions (10) as

\begin{tabular}{|c|c|c|c|}
\hline$\Gamma$ & $\kappa=0.0$ & $\kappa=0.2$ & $\kappa=0.4$ \\
\hline 1 & $-0.57173 \pm 0.01388$ & $-0.57866 \pm 0.01429$ & $-0.59912 \pm 0.01449$ \\
\hline 2 & $-0.65983 \pm 0.00800$ & $-0.66589 \pm 0.00785$ & $-0.68345 \pm 0.00775$ \\
\hline 5 & $-0.75101 \pm 0.00419$ & $-0.75677 \pm 0.00425$ & $-0.77038 \pm 0.00396$ \\
\hline 10 & $-0.79954 \pm 0.00235$ & $-0.80447 \pm 0.00250$ & $-0.81795 \pm 0.00235$ \\
\hline 20 & $-0.83340 \pm 0.00136$ & $-0.83783 \pm 0.00147$ & $-0.85100 \pm 0.00143$ \\
\hline 40 & $-0.85648 \pm 0.00078$ & $-0.86064 \pm 0.00086$ & $-0.87340 \pm 0.00078$ \\
\hline 60 & $-0.86595 \pm 0.00052$ & $-0.87029 \pm 0.00056$ & $-0.88290 \pm 0.00060$ \\
\hline 80 & $-0.87156 \pm 0.00046$ & $-0.87578 \pm 0.00047$ & $0.88841 \pm 0.00049$ \\
\hline 100 & $-0.87519 \pm 0.00035$ & $-0.87944 \pm 0.00041$ & $-0.89196 \pm 0.00033$ \\
\hline 120 & $-0.87786 \pm 0.00033$ & $-0.88200 \pm 0.00031$ & $-0.89444 \pm 0.00035$ \\
\hline 140 & $-0.87982 \pm 0.00031$ & $-0.88404 \pm 0.00029$ & $-0.89656 \pm 0.00029$ \\
\hline 160 & $-0.88561 \pm 0.00027$ & $-0.88930 \pm 0.00065$ & $-0.90216 \pm 0.00030$ \\
\hline 180 & $-0.88706 \pm 0.00021$ & $-0.89118 \pm 0.00023$ & $-0.90352 \pm 0.00019$ \\
\hline 200 & $-0.88800 \pm 0.00020$ & $-0.89214 \pm 0.00020$ & $-0.90449 \pm 0.00023$ \\
\hline 240 & $-0.88943 \pm 0.00016$ & $-0.89357 \pm 0.00014$ & $-0.90593 \pm 0.00015$ \\
\hline 300 & $-0.89078 \pm 0.00012$ & $-0.89492 \pm 0.00012$ & $-0.90729 \pm 0.00012$ \\
\hline 400 & $-0.89210 \pm 0.00009$ & $-0.89624 \pm 0.00009$ & $-0.90861 \pm 0.00012$ \\
\hline 500 & $-0.89288 \pm 0.00008$ & $-0.89702 \pm 0.00008$ & $-0.90939 \pm 0.00008$ \\
\hline 600 & $-0.89340 \pm 0.00007$ & $-0.89754 \pm 0.00007$ & $-0.90991 \pm 0.00007$ \\
\hline 800 & $-0.89404 \pm 0.00005$ & $-0.89818 \pm 0.00005$ & $-0.91055 \pm 0.00005$ \\
\hline 1000 & $-0.89442 \pm 0.00004$ & $-0.89856 \pm 0.00004$ & $-0.91093 \pm 0.00004$ \\
\hline$\infty$ & -0.89593 & -0.90007 & -0.91245 \\
\hline$\Gamma$ & $\kappa=0.6$ & $\kappa=0.8$ & \\
\hline 1 & & & $-0.73423 \pm 0.01155$ \\
\hline 2 & $-0.71215 \pm 0.00876$ & $-0.75145 \pm 0.00761$ & $-0.79866 \pm 0.00718$ \\
\hline 5 & $-0.79536 \pm 0.00380$ & $-0.82793 \pm 0.00395$ & $-0.87127 \pm 0.00396$ \\
\hline 10 & $-0.84051 \pm 0.00240$ & $-0.87192 \pm 0.002$ & $-0.91103 \pm 0$ \\
\hline 20 & $-0.87284 \pm 0.00136$ & $-0.90269 \pm 0.00145$ & $-0.94029 \pm 0.00143$ \\
\hline 40 & $-0.89471 \pm 0.00078$ & $-0.92372 \pm 0.00080$ & $-0.96063 \pm 0.00080$ \\
\hline 60 & $-0.90385 \pm 0.00061$ & $-0.93265 \pm 0.00060$ & $-0.96910 \pm 0.00059$ \\
\hline 80 & $-0.90907 \pm 0.0$ & $-0.93773 \pm 0.00043$ & $=0.97408 \pm 0.00049$ \\
\hline 100 & $-0.91261 \pm 0.00036$ & $-0.94126 \pm 0.00034$ & $-0.97735 \pm 0.00036$ \\
\hline 120 & $-0.91523 \pm 0.00032$ & $-0.94366 \pm 0.00033$ & $-0.97976 \pm 0.00030$ \\
\hline 140 & $-0.91718 \pm 0.00029$ & $-0.94556 \pm 0.00028$ & $-0.98156 \pm 0.00029$ \\
\hline 160 & $-0.91874 \pm 0.00027$ & $-0.94705 \pm 0.00025$ & $-0.98303 \pm 0.00025$ \\
\hline 180 & $-0.92377 \pm 0.00022$ & $-0.95173 \pm 0.00026$ & $-0.98423 \pm 0.00022$ \\
\hline 200 & $-0.92488 \pm 0.00021$ & $-0.95304 \pm 0.00020$ & $-0.98821 \pm 0.00030$ \\
\hline 240 & $-0.92634 \pm 0.00016$ & $-0.95451 \pm 0.00016$ & $-0.99008 \pm 0.00017$ \\
\hline 300 & $-0.92770 \pm 0.00012$ & $-0.95590 \pm 0.00011$ & $-0.99149 \pm 0.00015$ \\
\hline 400 & $-0.92903 \pm 0.00009$ & $-0.95723 \pm 0.00009$ & $-0.99284 \pm 0.00010$ \\
\hline 500 & $-0.92982 \pm 0.00007$ & $-0.95802 \pm 0.00008$ & $0.99364 \pm 0.00007$ \\
\hline 600 & $-0.93034 \pm 0.00006$ & $-0.95854 \pm 0.00007$ & $-0.99416 \pm 0.00006$ \\
\hline 800 & $-0.93098 \pm 0.00004$ & $-0.95919 \pm 0.00005$ & $-0.99480 \pm 0.00005$ \\
\hline 1000 & $-0.93136 \pm 0.00004$ & $-0.95957 \pm 0.00004$ & $-0.99519 \pm 0.00004$ \\
\hline$\infty$ & -0.93288 & thoring & $-0.990 / 1$ \\
\hline
\end{tabular}

TABLE II. Equilibrium excess energy per particle, $u / \Gamma(N=686)$.

$$
p=\frac{\kappa}{6} \frac{\partial f}{\partial \kappa}+\frac{\Gamma}{3} \frac{\partial f}{\partial \Gamma} \quad \text { and } \quad s=-f+\Gamma \frac{\partial f}{\partial \Gamma} .
$$

The function $p(\kappa, \Gamma)$ is the "equation of state" for the Yukawa system-its difference from unity describes the deviation from ideal-gas behavior (which is recovered in the limit $\Gamma \rightarrow 0)$. An accurate representation for $p(\kappa, \Gamma)$ is of practical interest in, for example, the formulation of macroscopic descriptions for the behavior of dust/plasma suspensions that can help in understanding and controlling particulate contamination in industrial process plasmas.

Simulations were run over a wide range of $\Gamma$ for each of the values $\kappa=0.0,0.2,0.4,0.6,0.8$, and 1.0 . The resulting time-averaged measurements of $u / \Gamma$-i.e., the excess energy per particle in units of $Q^{2} / 4 \pi \epsilon_{0} a$-as computed from (7) 
TABLE III. Madelung energies for bec and fcc Yukawa lattices.

\begin{tabular}{ccc}
\hline \hline$\kappa$ & $E(\mathrm{bcc})$ & $E(\mathrm{fcc})$ \\
\hline 0.0 & -0.895929 & -0.895873 \\
0.1 & -0.896966 & -0.896911 \\
0.2 & -0.900074 & -0.900020 \\
0.3 & -0.905240 & -0.905190 \\
0.4 & -0.912448 & -0.912402 \\
0.5 & -0.921671 & -0.921631 \\
0.6 & -0.932879 & -0.932844 \\
0.7 & -0.946032 & -0.946004 \\
0.8 & -0.961088 & -0.961067 \\
0.9 & -0.977997 & -0.977984 \\
1.0 & -0.996706 & -0.996701 \\
1.1 & -1.017158 & -1.017161 \\
1.2 & -1.039292 & -1.039302 \\
\hline \hline
\end{tabular}

and (8), are listed in Table II. Runs that melted to the fluid state are as follows: for $\kappa=0.0,0.2$, and 0.4 , all runs with $\Gamma<160$; for $\kappa=0.6$ and 0.8 ; all runs with $\Gamma<180$; and for $\kappa=1.0$, all runs with $\Gamma<200$.

We can determine the free energy $f(\kappa, \Gamma)$ from the simulation data by expressing it as an integral of the measured internal energy $u(\kappa, \Gamma)$ over $\Gamma$. In terms of the dimensionless quantities (11), the free energy becomes

$$
f=u-s,
$$

and making use of the relations (9) and (10) we have

$$
\frac{\partial f}{\partial \Gamma}=\frac{u(\kappa, \Gamma)}{\Gamma}
$$

Integrating from the appropriate limit $(\Gamma=0$ for the fluid phase, $\Gamma=\infty$ for the solid phase) then gives the desired free energy expressions. These are derived in Sec. III B and Sec. III C, based on least-squares-fitting formulas for $u(\kappa, \Gamma)$.

\section{A. The Madelung energy}

Our approach is based on assuming that the Yukawasystem excess energy has an analytic dependence on $\kappa$, in both the fluid and solid phases, and thus expanding the coefficients of established forms ${ }^{31}$ for $u$ in the OCP case as power series about $\kappa=0$. It is convenient to fit to the "thermal" component of the excess energy, defined by

$$
u_{\mathrm{th}}(\kappa, \Gamma)=u(\kappa, \Gamma)-u_{\infty}(\kappa),
$$

where $u_{\infty}(\kappa)$ represents the bcc Madelung energy per particle in units of $k T$. Table III gives the values

$$
E(\kappa)=\lim _{\Gamma \rightarrow \infty} \frac{u(\kappa, \Gamma)}{\Gamma},
$$

which represent the Madelung energy per particle in units of $Q^{2} / 4 \pi \epsilon_{0} a$, so that $u_{\infty}(\kappa)=E(\kappa) \Gamma$. The variation of $E(\kappa)$ is illustrated in Fig. 1. By least-squares fitting, we find that $E(\kappa)$ can be represented to an accuracy of $\sim 10^{-6}$ for $0 \leqslant \kappa \leqslant 1$ by the quartic polynomial

$$
\begin{aligned}
E(\kappa)= & -0.895929+0.000025 \kappa-0.103950 \kappa^{2} \\
& +0.000579 \kappa^{3}+0.002570 \kappa^{4} .
\end{aligned}
$$

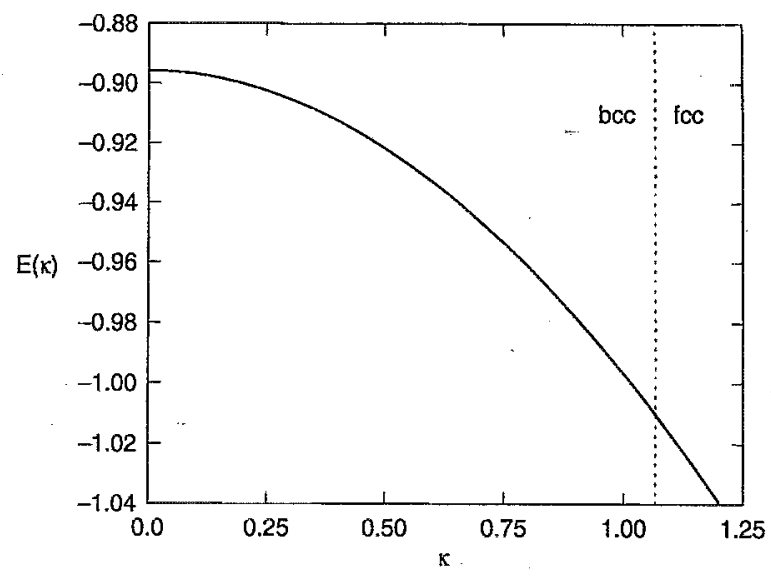

FIG. 1. Madelung energy per particle (in units of $Q^{2} / 4 \pi \epsilon_{0} a$; see Table III) of the Yukawa system as a function of $\kappa$. The bcc and fcc Madelung energies are indistinguishable on the scale of the graph; they cross at $\kappa \approx 1.066$.

\section{B. Free energy of the fluid phase}

For the fluid phase, we fit the measured excess energies to the expression

$$
u_{\mathrm{th}}(\kappa, \Gamma)=\delta a(\kappa) \Gamma+b(\kappa) \Gamma^{s}+c(\kappa)+d(\kappa) \Gamma^{-s}
$$

based on the variational hard sphere approach ${ }^{26}$ (which gives the exponent $s=1 / 4)$. The total excess energy is then $u=a \Gamma^{+}+b \Gamma^{s}+c+d \Gamma_{-}^{-s}$, where $a(\kappa)=E(\kappa)+\delta a(\kappa)$. Since the simulation data is rather sparse and of limited range, we take the dependence of the coefficients $\delta a, b, c, d$ in (16) on $\kappa$ to be no more than quadratic, i.e., we write

$$
\delta a(\kappa)=\delta a_{0}+\delta a_{1} \kappa+\delta a_{2} \kappa^{2},
$$

and similarly for $b(\kappa), c(\kappa)$, and $d(\kappa)$.

Twelve-term fits of the fluid data to expression (16) were performed for exponents $s=0.25,0.3,1 / 3,0.4$, and 0.5 . The value $s=1 / 3$ was found to give the best overall fit, with $\sigma=0.0041$ as the square root of the sample variance in $u_{\mathrm{th}}$. The coefficients $c_{2}$ and $d_{2}$ were poorly determined, however, and omitting these terms gives the ten-term fit (with $\sigma=0.0040$ ):

$$
\begin{aligned}
& \delta a_{0}=-0.003256 \pm 0.000121, \\
& \delta a_{1}=-0.000482 \pm 0.000267 \\
& \delta a_{2}=-0.000964 \pm 0.000176 \\
& b_{0}=-0.559934 \pm 0.006253 \\
& b_{1}=-0.026341 \pm 0.010536 \\
& b_{2}=-0.050644 \pm 0.003815 \\
& c_{0}=-0.192006 \pm 0.020737 \\
& c_{1}=-0.082556 \pm 0.032185 \\
& d_{0}=-0.040346 \pm 0.016406 \\
& d_{1}=-0.044115 \pm 0.025824
\end{aligned}
$$


TABLE IV. Excess energy per particle $u / \Gamma$ at small $\Gamma$ values $(N=500)$.

\begin{tabular}{ccccccc}
\hline \hline$\Gamma$ & $\kappa=0.0$ & $\kappa=0.2$ & $\kappa=0.4$ & $\kappa=0.6$ & $\kappa=0.8$ & $\kappa=1.0$ \\
\hline 0.00 & 0.0000 & -0.1000 & -0.2000 & -0.3000 & -0.4000 & -0.5000 \\
0.10 & -0.2652 & -0.2809 & -0.3294 & -0.3920 & -0.4750 & -0.5578 \\
0.20 & -0.3483 & -0.3600 & -0.3940 & -0.4520 & -0.5195 & -0.5964 \\
0.40 & -0.4431 & -0.4509 & -0.4794 & -0.5251 & -0.5799 & -0.6481 \\
0.60 & -0.4962 & -0.5088 & -0.5319 & -0.5732 & -0.6238 & -0.6846 \\
0.80 & -0.5406 & -0.5476 & -0.5708 & -0.6087 & -0.6561 & -0.7139 \\
1.00 & -0.5717 & -0.5787 & -0.5991 & -0.6317 & -0.6788 & -0.7342 \\
$f(\kappa, 1)$ & -0.4368 & -0.4495 & -0.4809 & -0.5284 & -0.5866 & -0.6541 \\
\hline \hline
\end{tabular}

Fitting to just OCP data gives values that agree well with the above: $\delta a=-0.003242, b=0.559336, \quad c=-0.189080$, $d=-0.044085$ (with $\sigma=0.0030$ ).

The coefficients of terms that are linear in $\kappa$ evidently have relatively large fractional uncertainties in the fit (17). Dropping these terms results in a six-parameter expression, with least-squares coefficients

$$
\begin{aligned}
& \delta a_{0}=-0.003584 \pm 0.000076, \\
& \delta a_{2}=-0.001050 \pm 0.000056, \\
& b_{0}=-0.576529 \pm 0.003890, \\
& b_{2}=-0.053157 \pm 0.001227, \\
& c_{0}=-0.240624 \pm 0.012969, \\
& d_{0}=-0.013147 \pm 0.010389
\end{aligned}
$$

that appear to be more precisely determined, although the quality of the fit is somewhat impaired $(\sigma=0.0047)$.

We write the excess free energy in the fluid phase as

$$
f(\kappa, \Gamma)=\int_{1}^{\Gamma} u\left(\kappa, \Gamma^{\prime}\right) \frac{\mathrm{d} \Gamma^{\prime}}{\Gamma^{\prime}}+f(\kappa, 1),
$$

where $f(\kappa, 1)$ is the free energy at $\Gamma=1$. Substituting from (16) then gives

$$
\begin{aligned}
f(\kappa, \Gamma)= & a(\kappa)(\Gamma-1)+b(\kappa) \frac{\Gamma^{s}-1}{s} \\
& +c(\kappa) \ln \Gamma-d(\kappa) \frac{\Gamma^{-s}-1}{s}+f(\kappa, 1) .
\end{aligned}
$$

For $\Gamma \gg 1$, the term $f(\kappa, 1)$ is a relatively minor contribution to the excess free energy. We estimate this term by performing a few $N=500$ runs from random initial configurations. The $u / \Gamma$ values obtained from these runs are given in Table IV (note that $u / \Gamma \rightarrow-\kappa / 2$, which represents the energy of the Debye sheaths, ${ }^{36}$ as $\Gamma \rightarrow 0$ ). For each $\kappa$, Table IV also gives a value for $f(\kappa, 1)$, as determined by a Simpson-rule quadrature. We find that the least-squares cubic

$f(\kappa, 1)=-0.4368-0.0105 \kappa-0.2801 \kappa^{2}+0.0733 \kappa^{3}$

reproduces the simulation data to nearly four decimal places. The OCP data in Table IV are in fair agreement with earlier studies. $^{27}$
To obtain the total free energy for the fluid phase, we must add the ideal-gas contribution $f_{0}(\kappa, \Gamma)$ to the excess free energy (19). From the expression for the entropy of an ideal $\operatorname{gas}^{37}$ we may write this in the form

$$
\begin{aligned}
f_{0}(\kappa, \Gamma)= & \ln \left[\left(\frac{2 \pi \hbar^{2}}{m k T}\right)^{3 / 2} n\right]-1 \\
= & 3 \ln \kappa+\frac{3}{2} \ln \Gamma-1+\ln \sqrt{\pi / 6} \\
& -3 \ln \frac{Q^{2}}{4 \pi \epsilon_{0} \hbar \omega_{\mathrm{p}} \lambda_{D}} .
\end{aligned}
$$

(Note that, in the final term, the product

$$
\omega_{\mathrm{p}} \lambda_{D}=\left[\left(\frac{q_{i}}{Q}\right)^{2}\left(\frac{\bar{n}_{i}}{n}\right) \frac{m}{k T_{i}}+\left(\frac{e}{Q}\right)^{2}\left(\frac{\bar{n}_{e}}{n}\right) \frac{m}{k T_{e}}\right]^{-1 / 2}
$$

of the particulate plasma frequency and the Debye length is independent of $\kappa$ and $\Gamma$ under the assumption that $\tilde{n}_{i} / n$, $\bar{n}_{e} / n$ are constants, and $T_{i}, T_{e}$ are unrelated to $T$.) Correspondingly, ideal-gas contributions $p_{0}(\kappa, \Gamma)=1$ and

$$
\begin{aligned}
s_{0}(\kappa, \Gamma)= & -3 \ln \kappa-\frac{3}{2} \ln \Gamma+\frac{5}{2}-\ln \sqrt{\pi / 6} \\
& +3 \ln \frac{Q^{2}}{4 \pi \epsilon_{0} \hbar \omega_{\mathrm{p}} \lambda_{D}}
\end{aligned}
$$

must be added to expressions (12) to obtain the total pressure and entropy in the fluid phase.

For finite $n$, the OCP limit $\kappa=0$ is realized as $\lambda_{D} \rightarrow \infty$. The singularity of the first term in expression (20) is thus cancelled by the last term, and in this limit the ideal-gas free energy may be written as: ${ }^{23}$

$$
f_{0}(0, \Gamma)=3 \ln \Gamma+\frac{3}{2} \ln (k T)_{\mathrm{Ry}}-1+\ln \frac{3 \sqrt{\pi}}{4}
$$

where $(k T)_{\text {Ry }}$ denotes $k T$ measured in Rydberg units, $\frac{1}{2}\left(Q^{2} / 4 \pi \epsilon_{0} \hbar\right)^{2} m$, for the particulates.

\section{Free energy of the solid phase}

For the solid phase, we expand the excess thermal energy as

$$
u_{\mathrm{th}}(\kappa, \Gamma)=\frac{3}{2}+\frac{A_{1}(\kappa)}{\Gamma}+\frac{A_{2}(\kappa)}{\Gamma^{2}}+\cdots,
$$


where $3 / 2$ is the harmonic component, and the power series in $\Gamma^{-1}$ represents the anharmonic terms. Again, we take the dependence of the coefficients of these terms on $K$ to be quadratic:

$$
A_{j}(\kappa)=A_{j 0}+A_{j 1} \kappa+A_{j 2} \kappa^{2} \quad j=1,2, \ldots .
$$

The lowest- $\Gamma$ runs that did not melt to the fluid state $(\Gamma=160$ for $\kappa=0.0,0.2$, and $0.4 ; \Gamma=180$ for $\kappa=0.6$ and 0.8 ; and $\Gamma=200$ for $\kappa=1.0$ ) produced conspicuously "noisy" energy values, and were therefore omitted from the least-squares fit to expression (21).

Keeping only the $\Gamma^{-1}$ and $\Gamma^{-2}$ terms in the expansion (21) for the solid phase, an unweighted fit to all six coefficients $A_{j k}$ for $j=1,2$ and $k=0,1,2$ was first attempted. While this gave reasonably good agreement with the data $(\sigma$ $=0.0024)$, the estimated uncertainties of the linear coefficients $A_{11}, A_{21}$ were large compared to their nominal values. Consequently, these terms were dropped and the resulting four-term fit gave

$$
\begin{array}{ll}
A_{10}=9.13 \pm 0.52, & A_{12}=4.37 \pm 1.16, \\
A_{20}=1526 \pm 117, & A_{22}=479 \pm 301,
\end{array}
$$

with $\sigma=0.0024$ again (the above values do not differ significantly from those obtained with the six-term fit-moreover, fitting only the OCP data gives $A_{10}=9.07 \pm 0.77$ and $A_{20}=1500 \pm 168$ with $\sigma=0.0021$ ).

The $A_{10}$ quoted above is in fair agreement with the OCP value of 10.645 (for $N=686$ ) predicted by thermodynamic perturbation theory ${ }^{38}$. Note, however, that coefficients obtained by fitting to simulation data are somewhat sensitive to the number of terms retained in (21) and the range of $\Gamma$ covered by the data [for $170 \leqslant \Gamma \leqslant 2000$, a previous OCP study $^{32}$ gave $A_{10}=8.19 \pm 0.20, A_{20}=1697 \pm 44$ for a twoterm fit, and $A_{10}=9.65 \pm 0.32, A_{20}=840 \pm 171, A_{30}$ $=(1.101 \pm 0.216) \times 10^{5}$ for a three-term fit].

In the classical limit, the free energy associated with harmonic lattice vibrations can be expressed ${ }^{24}$ for large $N$ as

$$
f_{\text {harm }}(\kappa, \Gamma)=E(\kappa) \Gamma+\frac{1}{N} \sum_{k=1}^{3 N-3} \ln \frac{\omega_{k}}{\omega_{\mathrm{p}}}+3 \ln \frac{\hbar \omega_{\mathrm{p}}}{k T},
$$

where the sum is taken over the $3 N-3$ normal-mode frequencies $\omega_{k}$ for the oscillation of a bcc lattice of $N$ particles. Denoting the $N \rightarrow \infty$ limit of this sum by $\Sigma(\kappa)$, and writing $\ln \left(\hbar \omega_{\mathrm{p}} / k T\right)=\ln \kappa+\ln \Gamma-\ln \left(Q^{2} / 4 \pi \epsilon_{0} \hbar \omega_{\mathrm{p}} \lambda_{D}\right)$, we obtain

$$
\begin{aligned}
f(\kappa, \Gamma)= & E(\kappa) \Gamma+\Sigma(\kappa)+3 \ln \kappa+3 \ln \Gamma \\
& -3 \ln \frac{Q^{2}}{4 \pi \epsilon_{0} \hbar \omega_{\mathrm{p}} \lambda_{D}}-\frac{A_{1}(\kappa)}{\Gamma}-\frac{A_{2}(\kappa)}{2 \Gamma^{2}}-\cdots
\end{aligned}
$$

as the total free energy for the solid phase. Here the contribution of the anharmonic terms in (21) has been included (assuming that the harmonic approximation becomes exact as $\Gamma \rightarrow \infty)$.

The eigenfrequencies $\omega_{k}$ of an $N$-particle bcc Yukawa lattice may, in principle, be computed using standard techniques, ${ }^{39}$ and the quantity $\Sigma(\kappa)$ can then be estimated for various $\kappa$ values by letting $N \rightarrow \infty$. However, since the

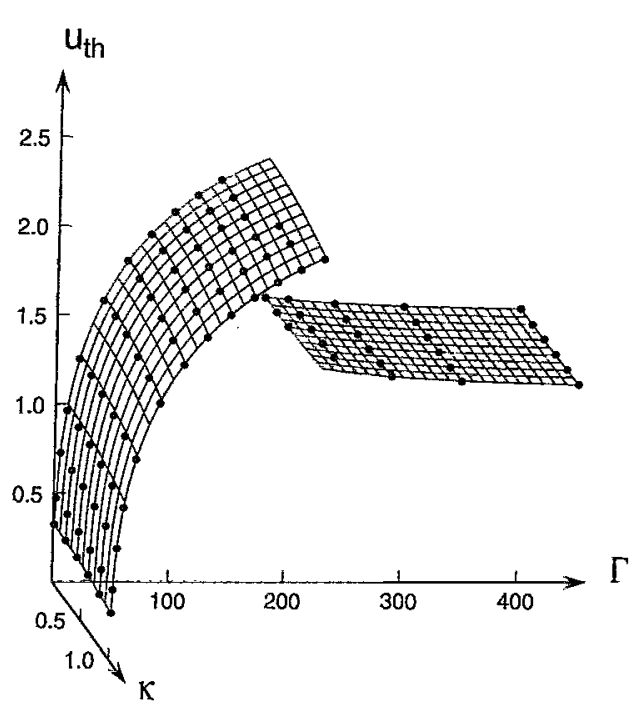

FIG. 2. Fitted thermal excess energy surfaces for fluid and solid phases of the Yukawa system, as defined by expressions (16) and (21) together with the coefficients (17) and (22). Also displayed are the simulation data. The fluid energy surface is shown for $1 \leqslant \Gamma \leqslant 180$ and the solid energy surface for $180 \leqslant \Gamma \leqslant 400$.

calculations are not trivial, and the "static" (Madelung) term $E(\kappa) \Gamma$ dominates the $\kappa$-dependence of expression (23), we shall be content at present to set the $\Sigma(\kappa)$ term identically equal to the OCP value $\Sigma(0)=-2.4938$ computed by Pollock and Hansen. ${ }^{24}$

In the OCP case $(\kappa=0)$ it is more convenient to re-write expression (23) in the nonsingular form

$$
\begin{aligned}
f(0, \Gamma)= & E(\kappa) \Gamma+\Sigma(\kappa)+\frac{9}{2} \ln \Gamma+\frac{3}{2} \ln (k T)_{\mathrm{Ry}} \\
& +\frac{3}{2} \ln \frac{3}{2}-\frac{A_{1}(\kappa)}{\Gamma}-\frac{A_{2}(\kappa)}{2 \Gamma^{2}}-\cdots
\end{aligned}
$$

\section{The phase transition}

The fitted thermal energy surfaces for the fluid and solid phases, as defined by expressions (16) and (21) and the coefficients (17) and (22), are shown together with the simulation data in Fig. 2. Near the phase transition, the fluid thermal energy surface curves slightly downward with increasing $\kappa$, while the solid thermal energy surface curves slightly upward. This may be seen more clearly in Fig. 3, which shows constant $-\Gamma$ contours on these surfaces. Note, however, that the variation in the static (Madelung) energy $E(\kappa)$ will dominate these mild trends.

The intersection of the derived free energy surfaces for the solid and fluid phases, given by expression (23) and the sum of expressions (19) and (20), respectively, was computed to determine the freezing/melting curve $\Gamma_{m}(\kappa)$ in the $(\kappa, \Gamma)$ plane. This is illustrated in Fig. 4.

For the OCP, we obtain $\Gamma_{m}(0) \approx 171$, in agreement with recent estimates. ${ }^{32,38,40}$ As $\kappa$ increases, $\Gamma_{m}$ exhibits a monotonic increase that becomes increasingly pronouncedrepresentative values are $\Gamma_{m} \approx 179,200,237,293$, and 378 


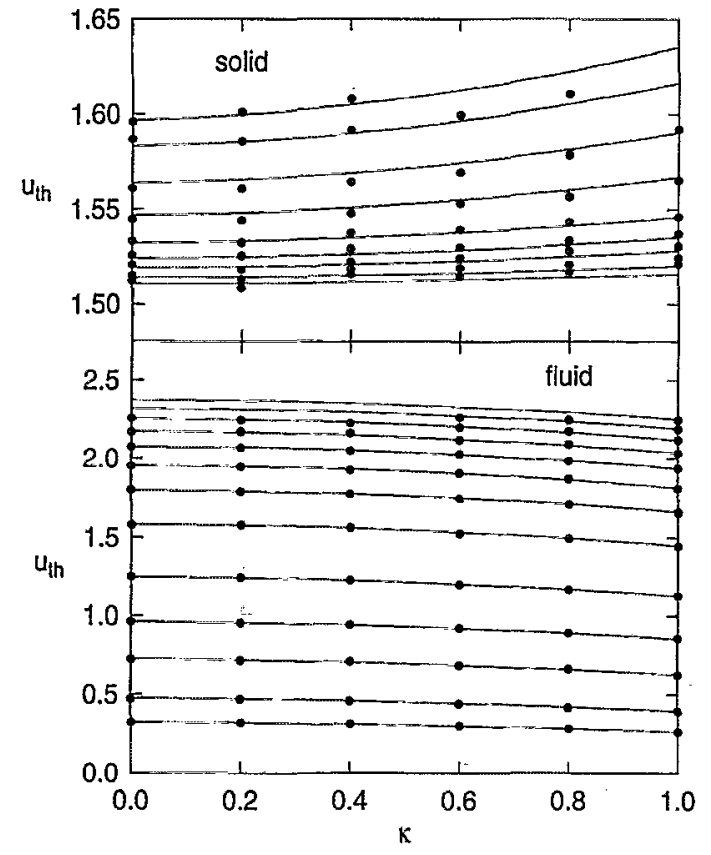

FIG. 3. Constant- $\Gamma$ contours on fitted thermal energy surfaces for the fluid and solid phases of the Yukawa system, together with simulation data. For the fluid phase, the lowermost contour is $\Gamma=1$ and the uppermost is $\Gamma=180$. For the solid phase, the contours range from $\Gamma=180$ to $\Gamma=1000$ (the solid data is shown on a greatly magnified scale).

for $\kappa=0.2,0.4,0.6,0.8$, and 1.0. Note that, for larger $\kappa$ values, the parameter $\Gamma$ is no longer a fair measure of the ratio of the particle kinetic energy to the pair potential energy. The quantity

$$
\Gamma^{*}=\Gamma \exp (-\kappa)
$$

is perhaps a better "coupling parameter" in this respect. The dotted curve in Fig. 4 shows the freezing/melting curve in terms of this quantity, indicating that the phase transition

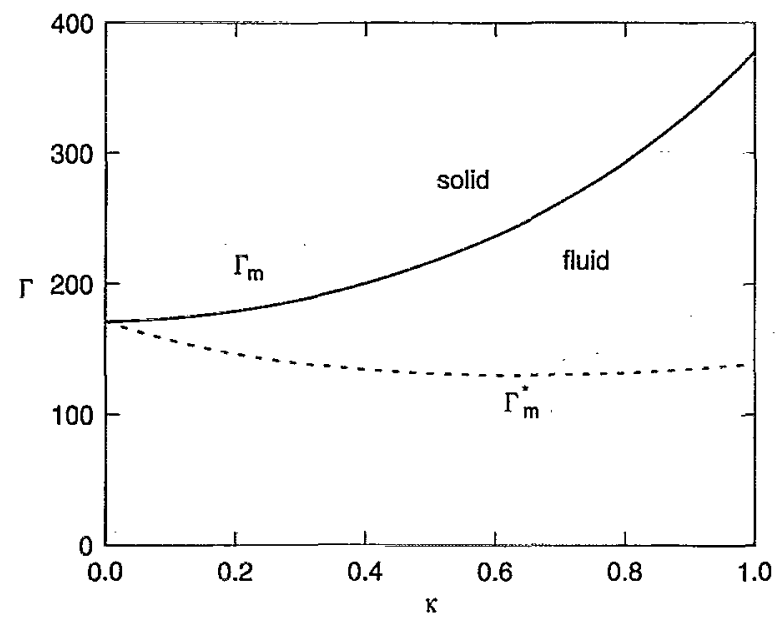

FIG. 4. The freezing/melting curve $\Gamma_{m}(\kappa)$, as determined by intersecting the solid and fiuid free energy surfaces obtained from the simulation data. The dashed curve represents $\Gamma_{m}^{*}(\kappa)$, where $\Gamma^{*}=\Gamma \exp (-\kappa)$. occurs at a reasonably constant coupling strength [there is perhaps a slight decline or dip in the $\Gamma_{m}^{*}(\kappa)$ curve; whether this is a genuine physical effect or an artifact of the fitting is unclear].

It is interesting to compare our results on the phase transition with those of Robbins, Kremer, and Grest. ${ }^{15}$ These authors represent the freezing/melting curve in the form

$$
\tilde{T}=\left(\frac{3}{4 \pi}\right)^{2 / 3} \frac{k T}{m a^{2} \omega_{E}^{2}}=0.00246+0.000274\left(\frac{4 \pi}{3}\right)^{1 / 3} \kappa,
$$

where $\omega_{E}$ is the Einstein frequency for a bcc lattice, and the fractional powers of $4 \pi / 3$ arise since $n^{-1 / 3}$, rather than $(4 \pi n / 3)^{-1 / 3}$, is used for $a$ in Ref. 15 . Note that the parameter $\lambda$ adopted in Ref. 15 is proportional to our $\kappa$, namely, $\kappa=(3 / 4 \pi)^{1 / 3} \lambda \approx 0.62035 \lambda$.

The formula (26) was based on an extrapolation of simulation data for $\kappa \geq 1$ to the OCP transition determined by Pollock and Hansen. ${ }^{24}$ The $\Gamma_{m}$ values it predicts are systematically somewhat lower than those shown in Fig. 4. For example, using the values of $(4 \pi / 3)^{2 / 3} m a^{2} \omega_{E}^{2}$ in units of $(3 / 4 \pi)^{1 / 3} Q^{2} / 4 \pi \epsilon_{0} a$ listed in Table I of Ref. 15 we find that $\Gamma_{m} \approx 168$ at $\lambda=1(\kappa=0.62)$ and $\Gamma_{m} \approx 228$ at $\lambda=2(\kappa=1.24)$, which may be compared with our present values $\Gamma_{m} \approx 293$ at $\kappa=0.6$ and $\Gamma_{m} \approx 378$ at $\kappa=1.0$.

Several factors may contribute to the observed discrepancy:

- No data for $0<\kappa<1$ was used in deducing the formula (26), and the Pollock and Hansen value ${ }^{24}$ $\Gamma_{m}=155$ adopted for $\kappa=0$ is too small according to recent OCP studies; ${ }^{32,38,40}$ one should therefore not expect accurate predictions from this formula when $\kappa$ $\leqslant 1$.

- The simulations in Ref. 15 employed only the "minimum-image method"-with a cutoff radius $r_{c}$ for particle interactions-to emulate infinite periodic boundary conditions, rather than the "effective" pair potential (4) with summation over all pairwise interactions; this can be expected to introduce some systematic error unless $\kappa \gg 1$.

- Equation (26) was based on the Lindemann criterion and observations of which runs melted from crystalline initial configurations, rather than the intersection of fitted free-energy surfaces as used here.

Overall, we believe that our results present a more accurate picture for the Yukawa-system phase transition in the regime of weak screening ( $\kappa \leqslant 1$ ), although the accuracy of the graph in Fig. 4 is perhaps suspect at the largest $\kappa$ values. The principal factors motivating this cautionary remark are: (i) concerns over the validity of expanding the OCP freeenergy coefficients as quadratic polynomials up to $\kappa=1$; (ii) our neglect of the $\kappa$-dependence of the $\Sigma(\kappa)$ term in the solid free energy (23); and (iii) relatively large uncertainties in the coefficients of the least-squares fitting formulas.

We note that, for $\kappa \leqslant 0.6$, only one or two runs with $\Gamma<\Gamma_{m}$ did not melt from a crystalline initial configuration within the duration of the simulation, whereas for $\kappa=0.8$ ( $\Gamma_{m}=293$ ) the $\Gamma=180,200$, and 240 runs did not melt 


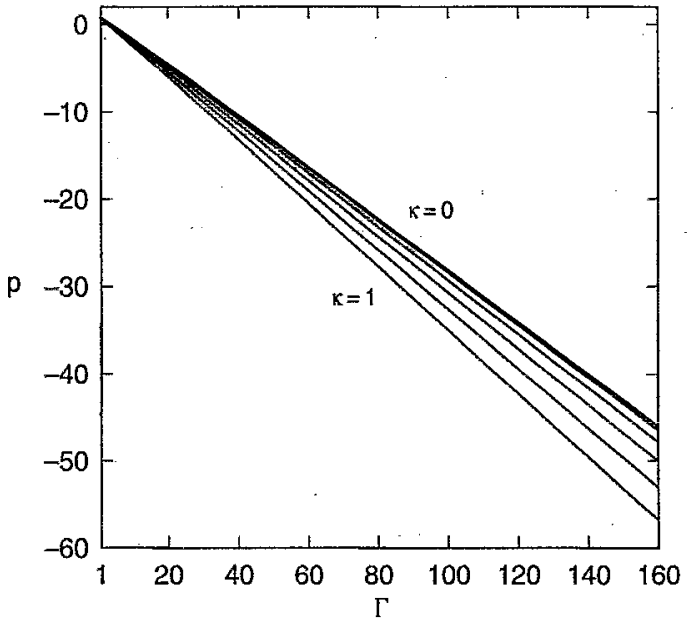

FIG. 5. Normalized pressure $p(\kappa, \Gamma)$ for the fluid phase of the Yukawa system as a function of $\Gamma$ for $\kappa$ values $0.0,0.2,0.4,0.6,0.8$, and 1.0 .

within the prescribed simulation time, and for $\kappa=1.0$ $\left(\Gamma_{m}=378\right)$ the $\Gamma=200,240$, and 300 cases had not melted before termination.

\section{E. Equation of state for the fluid phase}

In the context of particulate contamination in process plasmas, the equation of state for the fluid phase is of practical interest, since it provides a means of describing the transport of particulates on a simple continuum basis.

From Eqs. (19), (20), and (12) the fluid equation of state is given by

$$
\begin{aligned}
p(\kappa, \Gamma)=1 & +\frac{1}{3}\left[a \Gamma+b \Gamma^{s}+c+d \Gamma^{-s}\right] \\
& +\frac{\kappa}{6}\left[\frac{\mathrm{d} a}{\mathrm{~d} \kappa}(\Gamma-1)+\frac{\mathrm{d} b}{\mathrm{~d} \kappa} \frac{\Gamma^{s}-1}{s}+\frac{\mathrm{d} c}{\mathrm{~d} \kappa} \ln \Gamma\right. \\
& \left.-\frac{\mathrm{d} d}{\mathrm{~d} \kappa} \frac{\Gamma^{-s}-1}{s}+\frac{\mathrm{d} f(\kappa, 1)}{\mathrm{d} \kappa}\right]
\end{aligned}
$$

where $a=E+\delta a$, the coefficients of $E, \delta a, b, c, d$; and $f(\kappa, 1)$ as polynomials in $\kappa$ being given in Secs. III A and III $B$. Note that the above expression does not include the (ideal gas) pressure due to the homogeneous background plasma. This must be added to give the total pressure (for the OCP it becomes infinite if $\kappa \rightarrow 0$ at constant $n$, i.e., $\left.T_{i}, T_{e} \rightarrow \infty\right)$. We shall assume here that $T_{i}, T_{e}$ and $\bar{n}_{i} / n, \bar{n}_{e} / n$ are fixed, so that the term dependent on $\omega_{\mathrm{p}} \lambda_{D}$ in (20) is a constant, and changes in $\kappa$ and $\Gamma$ correspond to changes in the density and temperature of the particulates.

Several constant $-\kappa$ contours on the computed equationof-state surface are shown in Fig. 5. It is seen that, in the strongly-coupled regime $(\Gamma \geqslant 1)$, the pressure is less than the ideal gas value $p(\kappa, \Gamma)=1$ for all $\Gamma$ and becomes strongly negative as one approaches the phase transition.

For $\kappa \leqslant 1$, the presence of Debye screening does not appear to incur a pronounced departure from the behavior of the unscreened (OCP) system, at least in terms of the equa-

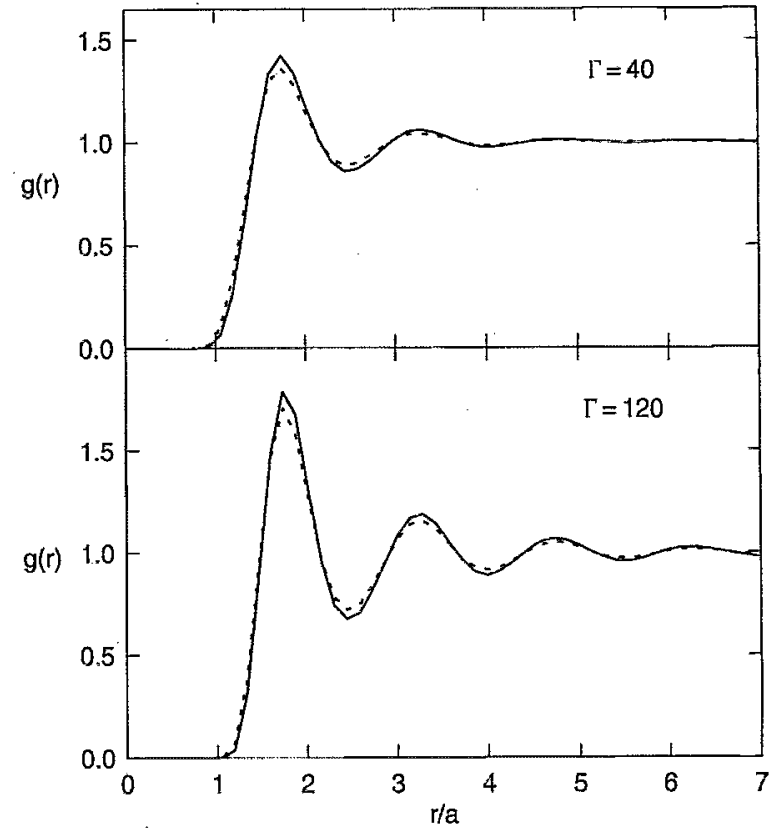

FIG. 6. Comparison of pair correlation functions for the OCP (solid curve) and $\kappa=1$ Yukawa system (dashed curve) obtained at $\Gamma=40$ and 120.

tion of state (see Fig. 5). This is also evident upon comparing the time-averaged correlation functions $g(r)$ obtained for $\kappa=0$ and $\kappa=1$, as shown in Fig. 6-the change is more significant, however, if one compares $g(r)$ at equal values of $\Gamma^{*}$ rather than $\Gamma$.

\section{CONCLUDING REMARKS}

We have presented a quantitative model for the thermodynamics of strongly-coupled systems of charged particles immersed in a responsive neutralizing background plasma, in the weakly-screened regime where the ratio $\kappa=a / \lambda_{D}$ of the interparticle spacing to the Debye length does not exceed unity. The model was constructed as an extrapolation of the extensively-studied case $\kappa=0$-the classical one-component plasma-corresponding to a strictly homogeneous background. Expressions for the Helmholtz free energy of the fluid and solid phases were derived, and in terms of the coupling parameter $\Gamma=Q^{2} / 4 \pi \epsilon_{0} a k T$ (where $Q$ and $T$ are the particle charge and temperature), the location of the phase transition was found to increase from $\Gamma_{m} \approx 171$ at $\kappa=0$ to $\Gamma_{m} \approx 378$ at $\kappa=1$. An equation of state for the fluid phase was also presented, suitable for use in continuum descriptions of the transport of charged particle suspensions, indicating a pressure somewhat below the ideal-gas value at $\Gamma=1$ and becoming strongly negative as $\Gamma \rightarrow \Gamma_{m}$.

\section{ACKNOWLEDGMENTS}

We are grateful to H. E. DeWitt, E. E. Salpeter, and Y. Rosenfeld for useful discussions.

${ }^{1}$ K. G. Spears, T. J. Robinson, and R. M. Roth, IEEE Trans. Plasma Sci. 14, 179 (1986).

${ }^{2}$ G. S. Selwyn, J. Singh, and R. S. Bennett, J. Vac. Sci. Technol. A 7, 2758 (1989). 
${ }^{3}$ G. S. Selwyn, J. Vac. Sci. Technol. B 9, 3487 (1991).

${ }^{4}$ R. N. Carlile, S. Geha, J. F. O'Hanlon, and J. C. Stewart, Appl. Phys. Lett. 59, 1167 (1991).

${ }^{5}$ H. Ikezi, Phys. Fluids 29, 1764 (1986).

${ }^{6}$ R. T. Farouki and S. Hamaguchi, Appl. Phys. Lett. 61, 2973 (1992).

${ }^{7}$ H. Thomas, G. E. Morfill, V. Demmel, J. Goree, B. Feuerbacher, and D. Möhlmann, Phys. Rev. Lett. 73, 652 (1994).

${ }^{8}$ Ordering and Organization in Ionic Solutions, edited by $\mathrm{N}$. Ise and $\mathrm{I}$. Sogami (World Scientific, Singapore, 1988).

${ }^{9}$ T. G. Northrop, Phys. Scripta 45, 475 (1992).

${ }^{10} \mathrm{M}$. Baus and J.-P. Hansen, Phys. Rep. 59, 1 (1980).

"Y. Rosenfeld, Phys. Rev. E 49, 4425 (1994).

${ }^{12}$ D. Hone, S. Alexander, P. M. Chaikin, and P. Pincus, J. Chem. Phys. 79, 1474 (1983).

${ }^{13}$ S. Alexander, P. M. Chaikin, P. Grant, G. J. Morales, P. Pincus, and D. Hone, J. Chem. Phys. 80, 5776 (1984).

${ }^{14}$ K. Kremer, M. O. Robbins, and G. S. Grest, Phys. Rev. Lett. 57, 2694 (1986).

${ }^{15}$ M. O. Robbins, K. Kremer, and G. S. Grest, J. Chem. Phys. 88, 3286 (1988).

${ }^{16}$ R. O. Rosenberg and D. Thirumalai, Phys. Rev. A 33, 4473 (1986).

${ }^{17}$ R. O. Rosenberg and D. Thirumalai, Phys. Rev. A 36, 5690 (1987).

${ }^{18}$ E. J. Meijer and D. Frenkel, J. Chem. Phys. 94, 2269 (1991).

${ }^{19}$ R. Ravelo, J. E. Hammerberg, and B. L. Holian, in Strongly Coupled Plasma Physics, edited by H. M. van Horn and S. Ichimaru (University of Rochester, Rochester, New York, 1993).

${ }^{20}$ N. Metropolis, A. W. Rosenbluth, M. N. Rosenbluth, A. H. Teller, and E. Teller, J. Chem. Phys. 21, 1087 (1953).
${ }^{21}$ R. T. Farouki and S. Hamaguchi, J. Comp. Phys. (in press).

${ }^{22}$ S. G. Brush, H. L. Sahlin, and E. Teller, J. Chem. Phys. 45, 2102 (1966).

${ }^{23}$ J.-P. Hansen, Phys. Rev. A 8, 3096 (1973).

${ }^{24}$ E. L. Pollock and J.-P. Hansen, Phys. Rev. A 8, 3110 (1973).

${ }^{25}$ H. E. DeWitt, Phys. Rev. A 14, 1290 (1976).

${ }^{26}$ H. E. DeWitt and Y. Rosenfeld, Phys. Lett. A 75, 79 (1979).

${ }^{27}$ W. L. Slattery, G. D. Doolen, and H. E. DeWitt, Phys. Rev. A 21, 2087 (1980).

${ }^{28}$ W. L. Slattery, G. D. Doolen, and H. E. DeWitt, Phys. Rev. A 26, 2255 (1982).

${ }^{29}$ H. L. Helfer, R. L. McCrory, and H. M. van Horn, J. Status Phys. 37, 577 (1984).

${ }^{30}$ S. Ogata and S. Ichimaru, Phys. Rev. A 36, 5451 (1987).

${ }^{31}$ G. C. Stringfellow, H. E. DeWitt, and W. L. Slattery, Phys. Rev. A 41, 1105 (1990)

${ }^{32}$ R. T. Farouki and S. Hamaguchi, Phys. Rev. E 47, 4330 (1993).

${ }^{33}$ J. M. Silva and B. J. Mokross, Phys. Rev. B 21, 2972 (1980).

${ }^{34}$ Y. Rosenfeld, Phys. Rev. A 46, 4922 (1992).

${ }^{35}$ Y. Rosenfeld, Phys. Rev. E 47, 2676 (1993).

${ }^{36}$ S. Hamaguchi and R. T. Farouki, J. Chem. Phys. 101, 9876 (1994).

${ }^{37}$ W. P. Allis and M. A. Herlin, Thermodynamics and Statistical Mechanics (McGraw-Hill, New York, 1952).

${ }^{38}$ D. H. E. Dubin, Phys. Rev. A 42, 4972 (1990).

${ }^{39}$ A. A. Maradudin, E. W. Montroll, G. H. Weiss, and I. P. Ipatova, Theory of Lattice Dynamics in the Harmonic Approximation, 2nd edition (Academic, New York, 1971).

${ }^{40}$ H. Nagara, Y. Nagata, and T. Nakamura, Phys. Rev. A 36, 1859 (1987). 